\title{
Erratum: CP-Violating Effect of the Th Nuclear Magnetic Quadrupole Moment: Accurate Many-Body Study of ThO [Phys. Rev. Lett. 113, 263006 (2014)]
}

\author{
L. V. Skripnikov, A. N. Petrov, A. V. Titov, and V. V. Flambaum
}

(Received 18 November 2015; published 15 December 2015)

DOI: 10.1103/PhysRevLett.115.259901

PACS numbers: 31.10.+z, 21.10.-k, 31.15.aj, 99.10.Cd

After the publication of this Letter, we have found an error in our computer code for the calculation of the matrix elements of the operator corresponding to the molecular parameter $W_{M}$ of the interaction of the nuclear magnetic quadrupole moment with electrons in heavy-atom molecules. This results in extra factor of 1.5 in the final value of $W_{M}$. Therefore, the last paragraph on page 263006-3 should read as follows: The final calculated value of $W_{M}$ is $1.10(8) \times 10^{33} \mathrm{~Hz} /\left(e \mathrm{~cm}^{2}\right)$. The theoretical uncertainty was estimated with a procedure similar to that given in Ref. [1]. We have found that the effect of spin polarization of subvalence closed shells contributes about $+0.06 \times 10^{33} \mathrm{~Hz} /\left(e \mathrm{~cm}^{2}\right)$, while the spin-orbit interaction of the valence and subvalence electrons contribute $-0.05 \times 10^{33} \mathrm{~Hz} /\left(e \mathrm{~cm}^{2}\right)$. The result of the earlier performed "semiempirical" estimate [2] $1.9 \times 10^{33} \mathrm{~Hz} /\left(e \mathrm{~cm}^{2}\right)$ is in reasonable agreement with the current ab initio calculations.

Equations (15) and (16) should read

$$
\begin{gathered}
0.16 W_{M} M=-7 \times 10^{10} \tilde{\theta} \times \mu \mathrm{Hz}, \\
0.16 W_{M} M=-4 \times \frac{10^{27}\left(\tilde{d}_{u}-\tilde{d}_{d}\right)}{\mathrm{cm}} \times \mu \mathrm{Hz} .
\end{gathered}
$$

The sentence after Eq. (26) should read as follows: The current limits on $|\tilde{\theta}|$ and $\left|\tilde{d}_{u}-\tilde{d}_{d}\right|\left(|\tilde{\theta}|<2.4 \times 10^{-10}\right.$, $\left|\tilde{d}_{u}-\tilde{d}_{d}\right|<6 \times 10^{-27} \mathrm{~cm}$; see Ref. [3]) correspond to the shifts $\left|0.16 W_{M} M\right|<17$ and $21 \mu \mathrm{Hz}$, respectively.

We thank Timo Fleig and Mikhail Kozlov for calling our attention to the problem with the $W_{M}$ value.

[1] L. V. Skripnikov and A. V. Titov, J. Chem. Phys. 142, 024301 (2015).

[2] V. V. Flambaum, D. DeMille, and M. G. Kozlov, Phys. Rev. Lett. 113, 103003 (2014).

[3] M. D. Swallows, T. H. Loftus, W. C. Griffith, B. R. Heckel, E. N. Fortson, and M. V. Romalis, Phys. Rev. A 87, 012102 (2013). 